Onkologe 2014 $\cdot 20: 267-267$

DOI 10.1007/s00761-014-2648-8

Online publiziert: 13. Februar 2014

(c) Springer-Verlag Berlin Heidelberg 2014

A.D. Hartkopf

Universitäts-Frauenklinik, Tübingen

\title{
Zirkulierende Tumor-DNA bei Patientinnen mit metastasiertem Mammakarzinom
}

\section{Originalpublikation}

Dawson SJ, Tsui DWY, Murtaza M et al (2013) Analysis of circulating tumor DNA to monitor metastatic breast cancer. N Engl J Med 368:1199-1209

\section{Hintergrund}

Brustkrebs kann im metastasierten Stadium nicht mehr kurativ behandelt werden. Umso wichtiger ist in dieser Situation die optimale Überwachung der Effektivität systemischer Therapien. Dies erfolgt in der Regel mittels radiologischer Verlaufskontrollen. Von entscheidendem Nachteil sind jedoch die relativ geringe Sensitivität und das notwendige zeitliche Intervall zwischen Beginn einer systemischen Therapie und der Kontrolle des Therapieerfolgs mittels Bildgebung. Vor diesem Hintergrund helfen Biomarker, schneller und genauer das Ansprechen einer Systemtherapie aufzuzeigen.

Ein vielversprechendes Tool zur Überwachung der Therapieeffektivität stellen zirkulierende Tumorzellen (ZTZ) dar. Werden mittels des FDA-zugelassenen CellSearch $^{\oplus}$ Systems (Veridex) mehr als 5 ZTZ in 7,5 ml Blut detektiert, so haben die betroffenen Patientinnen ein deutlich verschlechtertes Outcome [1]. Diese prognostische Wertigkeit findet sich sowohl zu Beginn einer neuen Therapie als auch zu unterschiedlichen Zeitpunkten während der Therapie. In einer vor kurzem auf dem San Antonio Breast Cancer Symposium vorgestellten prospektiv-randomisierten Studie (SWOG) wurde die prognostische Bedeutung von ZTZ bestätigt. Allerdings zeigten sich keine Implikationen für einen Therapiewechsel bei persistierend hohen ZTZ während einer systemischen Therapie [2].

Moderne Sequenzierungstechniken erlauben eine zunehmend ökonomische- re Identifikation genetischer Veränderungen von individuellen Tumoren. Weiterhin finden sich im Blutplasma zirkulierende DNA-Fragmente, die tumorspezifische Mutationen aufweisen. Entsprechend lassen sich patientenindividuelle Testverfahren designen, die auf Basis der tumorspezifischen Sequenzalterationen zirkulierende Tumor-DNA im Blutplasma detektieren.

\section{Studiendesign}

In einer prospektiven unizentrischen Studie untersuchten Dawson et al. die Bedeutung zirkulierender Tumor-DNA für die Überwachung von Patientinnen mit metastasiertem Brustkrebs. Sie Verglichen ihre Ergebnisse mit ZTZ, CA 15-3 und konventioneller Bildgebung. Hierfür wurde 53 Patientinnen mit metastasiertem Brustkrebs, bei denen Primärtumorgewebe verfügbar war, mehrfach Blut entnommen.

\section{Ergebnisse}

Bei 30 Patientinnen konnten geeignete genetische Alterationen im Primärtumormaterial detektiert werden. Zirkulierende Tumor-DNA wurde in 29 dieser 30 Patientinnen gefunden (97\%). CA 15-3 war in 2 von 27 Patientinnen erhöht (78\%) und ZTZ wurden in 26 von 30 Patientinnen (87\%) detektiert. Die Menge der detektierten Tumor-DNA war mit dem Gesamtüberleben, mit den CA-15-3-Serum-Spiegeln und mit der Anzahl der detektierten ZTZ vergesellschaftet. Weiterhin korrelierte im Rahmen einer seriellen Quantifizierung die Änderung der DNAMenge mit dem Therapieansprechen in der radiologischen Verlaufskontrolle.

Im Vergleich zu ZTZ und CA 15-3 wies die Menge der zirkulierenden Tumor-DNA eine größere Bandbreite auf und konnte die bestehende Tumorlast besser wiedergeben. Zudem konnten 97\% der Patientinnen durch die vorgestellte Methode erfasst werden, während es bei der ZTZ-Detektion nur $87 \%$ und bei der CA-15-3-Bestimmung nur $78 \%$ aller Patientinnen waren.

\section{Kommentar}

Die Arbeit von Dawson et al. ist als Proofof-Principle-Analyse zu verstehen, welche die hohe Sensitivität zirkulierender Tumor-DNA als Biomarker für Patientinnen mit metastasiertem Brustkrebs hervorhebt. Durch die Verwendung freier DNA im Plasma im Sinne einer „liquid biopsy“ ergeben sich enorme Möglichkeiten, nicht nur für die Implementierung neuer Tumormarker, sondern ebenso für das Verständnis von Tumorprogression und in Konsequenz für die Entwicklung neuer zielgerichteter Therapien.

\section{Korrespondenzadresse}

\section{Dr. A.D. Hartkopf}

Universitäts-Frauenklinik

Calwerstraße 7, 72076 Tübingen

andreas.hartkopf@med.uni-tuebingen.de

\section{Ethische Richtlinien}

Interessenkonflikt. A.D. Hartkopf gibt an, dass kein Interessenkonflikt besteht.

\section{Literatur}

1. Cristofanilli M et al (2004) Circulating tumor cells, disease progression, and survival in metastatic breast cancer. N Engl J Med 351:781-791

2. Smerage JB et al (2013) SWOG S0500 - A randomized phase III trial to test the strategy of changing therapy versus maintaining therapy for metastatic breast cancer patients who have elevated circulating tumor cell (CTC) levels at first follow-up assessment. San Antonio Breast Cancer Symposium (Abstract S5-03) 